\title{
Development of a Finger Pad Force Display for a Hand Haptic Interface
}

\author{
Haruhisa Kawasaki, Shinya Koide, Tetuya Mouri and Takahiro Endo \\ Gifu University, \\ Japan
}

\section{Introduction}

Most human interfaces in virtual environments use information of visual sensation and auditory sensation. By adding tactile sensation to these human interfaces, the human operator can handle objects with a realistic tactile sense and can perform complex tasks in the virtual reality environment. Haptic interfaces that present force and tactile feeling to the fingertips of a human operator have been investigated extensively over the last two decades [1]-[12]. Although some of these interfaces [9]-[12] provide three-dimensional force feeling to the human fingertip, few haptic interfaces cover the workspace of the human arm. These interfaces are classified into two types: wearable type interfaces [9], which are mounted firmly to the human hand, and opposed type interfaces [12], which are mounted in a position opposed to the human hand. These interfaces apply three-dimensional (3D) forces only to the human fingertips.

Medical doctors must use palpation in examining patients, and the force and tactile sensation on both the fingertips and the finger pads are important in such palpation. However, training doctors to perform palpation is difficult, because this requires the cooperation of the patient. Thus a breast palpation training system in a virtual reality environment [13] would be useful as a training tool for palpation. However, due to the limitation of human finger length, developing a haptic interface that displays 3D force feeling to both the fingertips and the finger pads is not easy.

The density of tactile sense organs in the human finger [14] is high in the fingertip and low in the finger pad. Hence, the human finger has a high sensitivity to 3D force at the fingertip but a low sensitivity at the finger pad. This suggests that a haptic interface that consists of 1D finger pad force display devices and a 3D fingertip force device would be effective for use in a virtual environment such as a virtual breast palpation training system.

The present paper describes a hand haptic interface for use in a virtual training system in which not only fingertip force display but also finger pad force display is required. The hand haptic interface consists of novel finger pad force display devices and a 3D fingertip haptic interface, known as HIRO II [12], which was developed by our group. The developed finger pad force display device is driven by a flat-type brushless DC motor and is easy attachable to the finger pad. The applied force is controlled by a time interval control, which is an open-loop control. Here we present the design concept of the hand haptic interface, the control method and specifications of the finger pad force display device, and the results of an experimental evaluation of manipulating a virtual object. We also provide a comparative 
evaluation between a case of using one force finger pad force display device and a case of using two force finger pad force display devices on an index finger when touching a rotating polyhedron.

\section{Hand haptic interface}

Humans manipulate objects using force and tactile feeling at the fingertip and the finger pad. For example, medical doctors search for tumors during breast palpation and manipulate internal organs during surgery using the fingertips and finger pads. To practice such medical procedures in a virtual environment, a hand haptic interface is required to apply forces to both the fingertips and finger pads.

The density of tactile sensory organs in the human finger is high in the fingertip and relatively low in the finger pad [14]. Hence, the human hand has a high sensitivity to 3D force at the fingertip [15] but a relatively low sensitivity at the finger pad. This suggests that a hand haptic interface that applies 3D force feeling to the finger tip and 1D force feeling to the finger pad would be effective for palpation training in a virtual reality environment, as shown in Fig. 1. In this figure, the human fingertip shows 3D force from the 3D fingertip force display device and the human finger pad shows 1D force, the direction of which is normal with respect to the surface of finger pad, from the 1D finger pad display device. The finger pad force display devices are attached to the proximal phalanges of the thumb and fingers and to the middle phalanges of the fingers. The total number of displayed points of the finger pads is nine.

The multi-fingered haptic interface robot HIRO II [12] shown in Fig. 2 is used to apply 3D fingertip forces. The haptic interface is described in detail in a previous report [8]. The mechanism of the haptic interface is outlined briefly herein to clarify the proposed hand haptic interface.

HIRO II can present force and tactile feeling at the five fingertips of the human hand. HIRO II is designed to be completely safe and is similar to the human upper limb both in shape and mobility. The mechanism of HIRO II consists of a six-degrees-of freedom (DOF) arm and a 15-DOF hand with a thumb and four fingers. Each finger has three joints, allowing three DOF. The first joint, relative to the base of the hand, allows abduction/adduction. The second joint and the third joint allow flexion/extension. The thumb is similar to the fingers except for the reduction gear ratio and the movable ranges of joint 1 and joint 2 . To read the finger loading, a six-axis force sensor is installed in the second link of each finger. The user must wear finger holders over his/her fingertips to manipulate the haptic interface. Each finger holder has a ball attached to a permanent magnet at the force sensor tip and forms a passive spherical joint. This passive spherical joint has two roles. First, it adjusts for differences between the human finger orientation and the haptic finger orientation. Second, the operator is able to remove his/her fingers from the haptic interface in case of a malfunction. The suction force generated by the permanent magnet is $5 \mathrm{~N}$.

\section{Finger pad haptic display device}

A. Mechanical design

A haptic device for the finger pad should be small and lightweight, so that it can be attached to the finger pad. The magnitude of the applied force must be sufficiently large, so that it can present realistic sensations during virtual object manipulation. The device should be easy to wear and should not obstruct the movement of the hand. Previously developed 
haptic devices for the finger tips or finger pads [16]-[19] have been problematic in that forces could not be applied to two finger pads of a finger simultaneously because of the size of the device or because the applied forces were insufficient. Therefore, we developed a novel finger pad force display device, as shown in Fig. 3(1), which can be attached to a finger at two points. This finger pad force display device consists of a body and a hook-and-loop fastener, which is wrapped around the finger pad. The mechanical structure of the device is shown in Fig. 3(2). The finger pad force display device is driven by a flat-type brushless DC motor (EC10, Maxon Motor) with a maximum torque of $0.176 \mathrm{mNm}$, a maximum rotational velocity of 22,000 rpm, a mass of $0.81 \mathrm{~g}$, and a diameter of $10 \mathrm{~mm}$. Reduction gears with a reduction ratio of 3.67 are attached to the rotor of the motor, and a screw mechanism with a pitch of $0.5 \mathrm{~mm}$ is attached to the output gear axis of the reduction gears. The nut of the screw mechanism moves up and down with the rotation of the motor with a range of movement of $4 \mathrm{~mm}$ and contacts the finger pad. The mass and output force are $4.8 \mathrm{~g}$ and 2.6 $\mathrm{N}$, respectively. Note that, in order to realize compactness and light weight, the finger pad force display device does not contain a force sensor. Hence, the contact force is open-loop controlled.

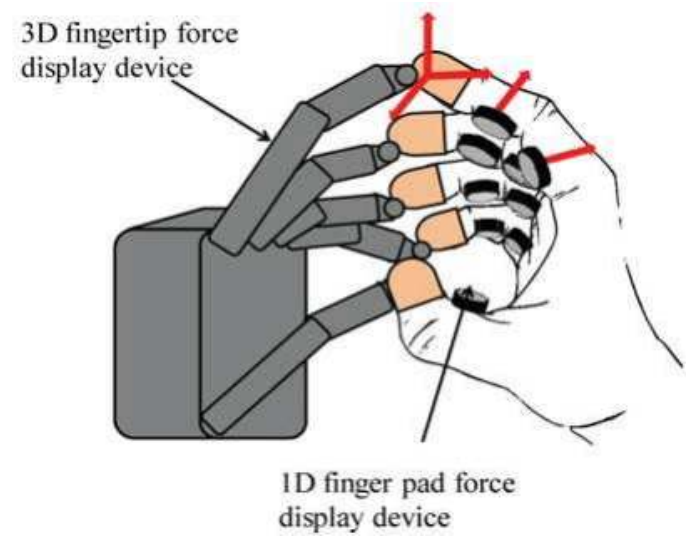

Fig. 1. Concept of proposed hand haptic interface

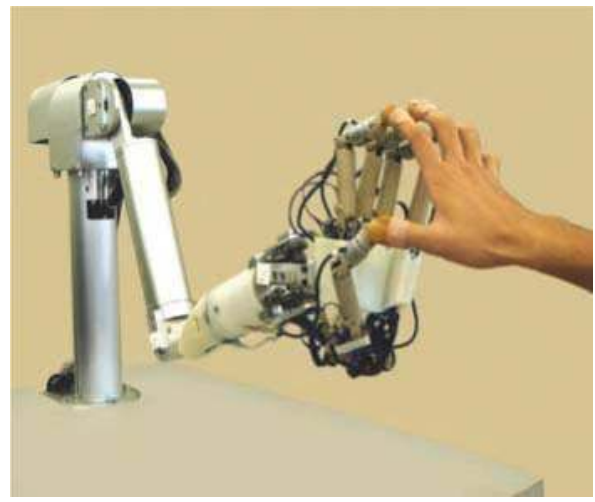

Fig. 2. Multi-fingered haptic interface robot HIRO II 


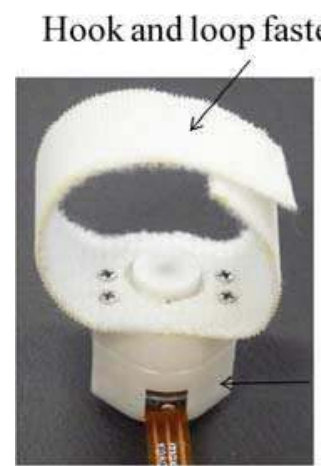

(1) Photograph
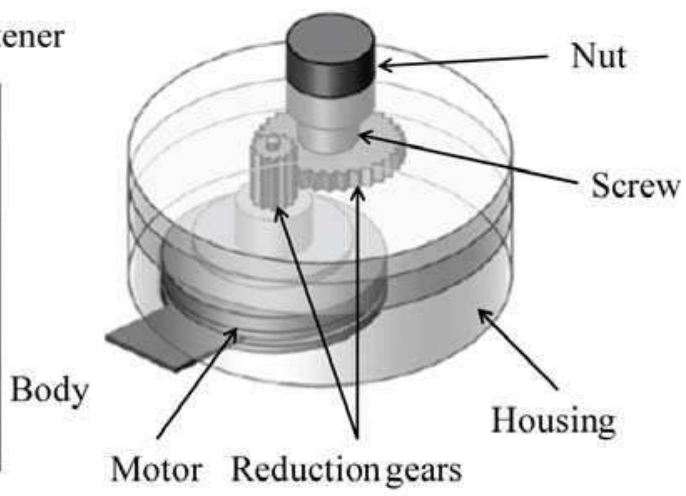

(2) Structure of body

Fig. 3. Developed finger pad force display

\section{B. Measurements of the displayed force}

To determine force on the finger pad of the human hand, we measure the force responses on a rigid plate and a gel plate [20] (Exseal Co.). The measurement system for the case of the gel plate is shown in Fig. 4. The force is measured by means of a six-axis force sensor (NANO sensor, Nitta Co.). The gel plate, which has a thickness of $5 \mathrm{~mm}$ and a hardness of 5 , as measured using an ASKER durometer (Type C) [20], is wedged between the sensor and the force display device. We selected this plate because it has a hardness that is similar to that of human skin. Fig. 4(1) shows the initial state, and Fig. 4(2) shows the end state after applying a force.

The step responses of the measured force are shown in Fig. 5. The displayed forces are 2.26 $\mathrm{N}$ for the rigid plate and $0.71 \mathrm{~N}$ for the gel plate, which is approximately $31 \%$ of the rigid plate case. This reduction is caused by the deformation of the gel plate, as shown in Fig. 4(2). A similar phenomenon will likely occur in the case of the finger pad of the human hand.

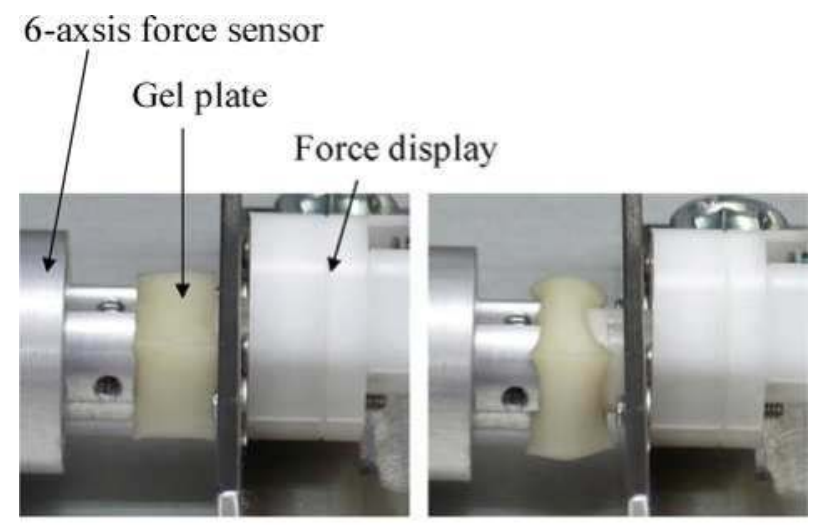
(1) Initial state
(2) End state after pushing

Fig. 4. Force measurement system 


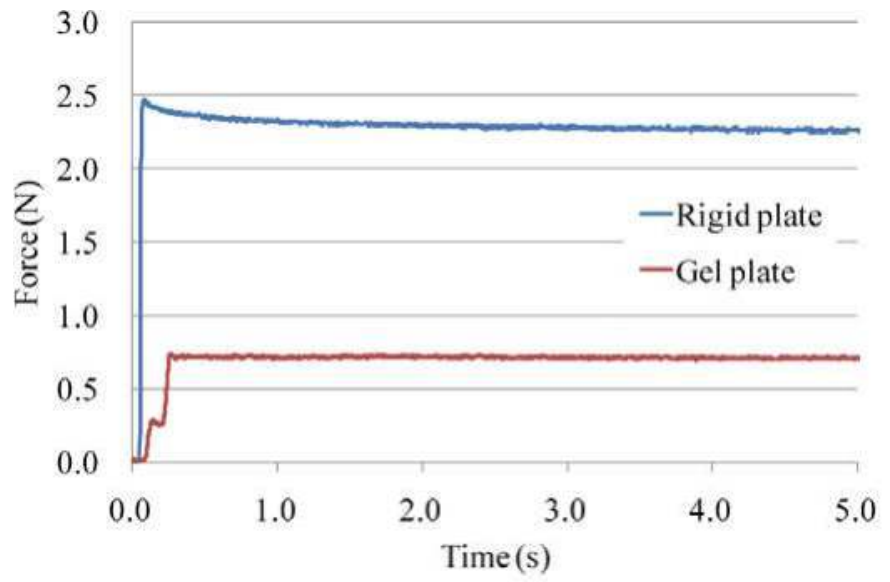

Fig. 5. Force responses by step input in case of rigid and gel plates

\section{Time interval control}

We adopt a time interval control in which the time interval of the input is controlled to adjust the applied force, because the finger pad force display device is not equipped with a force sensor. To confirm the effectiveness of the time interval control, we set the time interval of the input from $0.1 \mathrm{~s}$ to $0.5 \mathrm{~s}$ with intervals of $0.1 \mathrm{~s}$, which yields five levels of applied force. The experimental results are shown in Fig. 6. The root-mean-square of the measured forces at each time interval control are $0.13,0.26,0.39,0.56$, and $0.71 \mathrm{~N}$ with standard deviations of $0.013,0.01,0.011,0.013$, and $0.011 \mathrm{~N}$, respectively. Input of a longer time interval results in a higher level of force. These results reveal that the developed fingertip force display device can present a stepwise force to the finger pad of the human hand.

D. Experiment testing force sensation at the finger pad

We examined whether the human hand can distinguish the five levels of force applied by the finger pad force display device after nominal force (level 3) was applied. Ten subjects in their twenties, including eight male subjects and two female subjects, all right handed, tested the device. Each subject wore the finger pad force display device on the middle phalanx of the index finger and was asked to indicate whether the applied force was 'strong', 'the same as', 'weak', or 'unknown' compared to the nominal stimulus of level 3. The nominal stimulus and the comparison stimulus were presented alternately. The level of the comparison stimulus, i.e., from level 1 to level 5, was randomized, and the number of times each level of the comparison stimulus was presented was set to be 10 times. The results of the experiment are shown in Table I. When the difference between the nominal stimulus and the comparison stimulus was two levels, the correct answer rate was $98 \%$. However, the correct answer rate was reduced to $62 \sim 78 \%$ when the difference between the nominal stimulus and the comparison stimulus was at the zero or one level. Note that there are significant differences between individuals. One of the subjects answered weak for $90 \%$ of the comparison stimuli, whereas another subject answered weak for only $10 \%$ of the comparison stimuli.

Next, we examined whether the human hand could distinguish the force level when the nominal force level had not been applied. The subjects were the same as those of the 
previous experiment. First, subjects were presented with five forces, from level 1 to level 5 . The subjects were then presented with a comparison stimulus of a random level and asked to answer two questions. First, the subjects were asked to state the force level (levels 1 through 5, or unknown) of the presented stimulus. Second, the subjects were asked to state whether the force level of the stimulus was small, the same as, large, or unknown as compared to the previously applied stimulus. The results are shown in Table II. The average correct answer rate for the first question was $49.6 \%$, with a standard deviation of $17.6 \%$, and that for the second question was $84.4 \%$, with a standard deviation of $13.4 \%$. Although there were individual differences in the correct answer rate, we can say that the correct answer rate of the exact force level was not high and that the correct answer rate for the differences between the comparative stimuli was relatively high.

The results indicate that the stepwise force display device with the five-level time interval control can produce a recognizable force sensation.

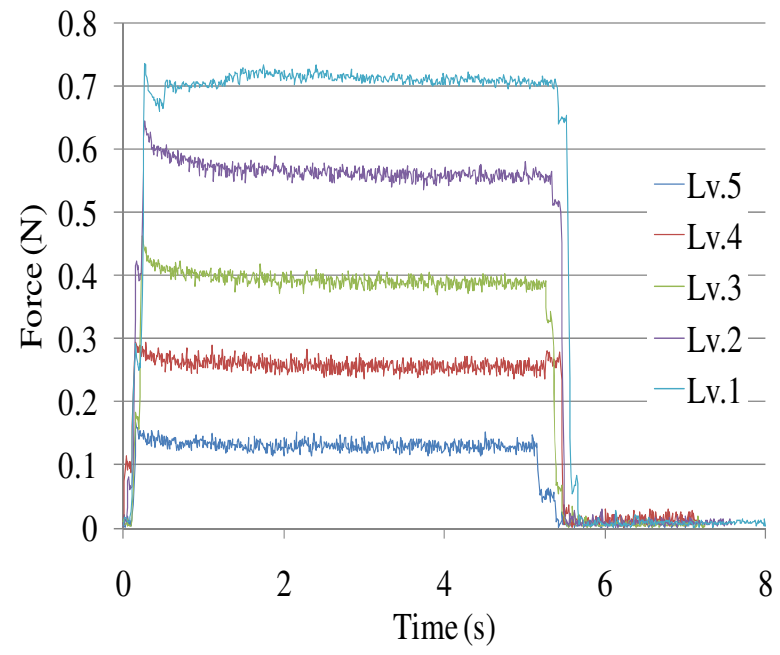

Fig. 6. Force responses by the time interval control in case of the gel plate

\begin{tabular}{|c|c|c|c|c|c|}
\hline $\begin{array}{c}\text { Displayed force } \\
\text { level }\end{array}$ & Weak & Same & Strong & Unclear & $\begin{array}{c}\text { Correct answer } \\
\text { rate [\%] }\end{array}$ \\
\hline 1 & 98 & 2 & 0 & 0 & 98 \\
\hline 2 & 62 & 35 & 3 & 0 & 62 \\
\hline 3 & 2 & 74 & 22 & 2 & 74 \\
\hline 4 & 2 & 16 & 78 & 4 & 78 \\
\hline 5 & 0 & 2 & 98 & 0 & 98 \\
\hline
\end{tabular}

Note: Each subject wore the finger pad force display device on the middle phalanx of the index finger and was asked to indicate whether the applied force was 'strong', 'the same as', 'weak', or 'unknown' compared to the nominal stimulus of level 3.

Table 1. Correct Answer rates for Comparisons with Nominal Stimulus 


\begin{tabular}{|c|c|c|c|c|c|c|c|c|c|c|}
\hline Subject No. & 1 & 2 & 3 & 4 & 5 & 6 & 7 & 8 & 9 & 10 \\
\hline $\begin{array}{c}\text { First } \\
\text { question }\end{array}$ & 32 & 20 & 40 & 68 & 60 & 40 & 68 & 72 & 56 & 40 \\
\hline $\begin{array}{c}\text { Second } \\
\text { question }\end{array}$ & 60 & 76 & 68 & 84 & 100 & 92 & 92 & 92 & 100 & 80 \\
\hline
\end{tabular}

Note: Subjects were presented with five forces, from level 1 to level 5 . Then, they were presented with a comparison stimulus of a random level and asked to answer two questions. First, the subjects were asked to state the force level (levels 1 through 5, or unknown) of the presented stimulus. Second, the subjects were asked to state whether the force level of the stimulus was small, the same as, large, or unknown as compared to the previously applied stimulus.

Table 2. Results Of Correct Answer rates

\section{Virtual reality system}

We have developed a hand haptic interface that incorporates a 3D fingertip force display device (HIRO II) and 1D finger pad force display devices, as shown in Fig. 7. The hand orientation of the operator is measured by a 3D orientation-tracking sensor (InertiaCube made by InterSense, Inc.), which is mounted on the back of the operator's hand. The hand position is measured by a 3D position-tracking sensor (Optorack Certus, Northern Digital Inc.). The operator wears five finger holders over the fingertips, for the connection with HIRO II, and nine finger pad force display devices on the middle phalanges of the fingers and the proximal phalanges of the thumb and fingers, as shown in Fig. 8.

The finger joint angles of the operator's hand are calculated using the inverse kinematics of the finger based on the positions of fingertips, measured by HIRO II, and the wrist point, measured by the 3D position tracking sensor. The solution of the inverse kinematics is not unique, because the human finger has three joints (distal interphalangeal joint, proximal interphalangeal joint, and metacarpophalangeal joint), each of which has four DOF. However, the fourth (distal interphalangeal) joint angle, $q_{4}^{i}$, depends on the third (proximal interphalangeal) joint angle, $q_{3}^{i}$, and can be approximated as $q_{3}^{i}=q_{4}^{i}$ [21], where the index $i$ indicates the order of the fingers. Using this relation, the inverse kinematics has a unique solution. The results of the calculations are shown in Fig. 9, in which the large balls indicate the fingertips and the small balls indicate the locations of the finger pad force display devices.

The control system of the hand haptic interface consists of a haptic interface control PC (HPC) and a virtual environment modeling PC (VPC). The constrained forces at the contact points between the hand and the virtual object are computed in the VPC and are sent to the HPC with a sampling cycle of $1 \mathrm{~ms}$ through a local LAN. HIRO II is controlled by a hybrid control comprising a finger force control and an arm position control, in which the haptic hand attitude is controlled such that the hand manipulability is optimized in real time [12]. The finger pad force display device is controlled by the time interval control with five levels.

\section{Experimental evaluation}

We examined the effectiveness of the haptic interface through two psychological tests. One involved grasping a cylindrical virtual object and the other involved touching a rotating 
polyhedron. Ten subjects in their twenties, including nine male subjects and one female subject, all right handed, participated in the evaluation.

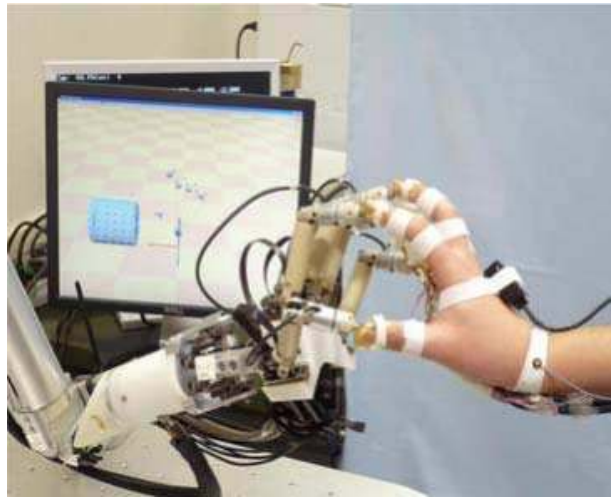

Fig. 7. Developed hand haptic interface

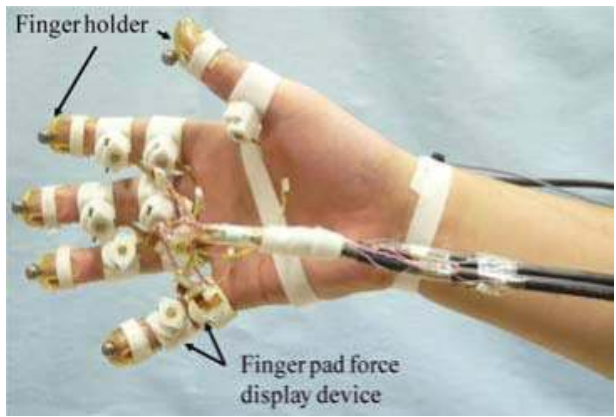

Fig. 8. Layout of the finger holders and the finger pad force display devices

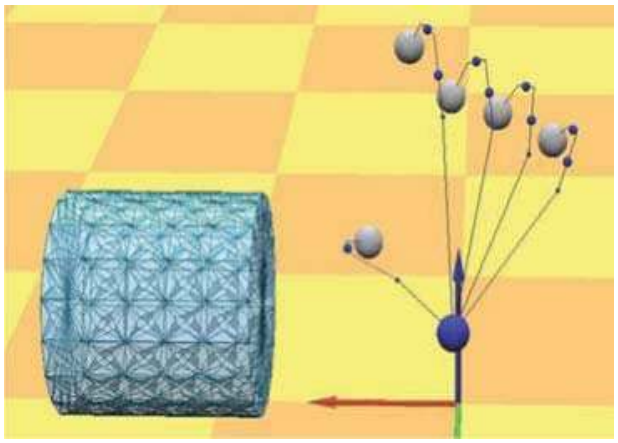

Fig. 9. Human hand model in computer graphics

A. Grasping of a cylindrical object.

In the experiment, the subjects were asked to grasp a virtual cylindrical object with an enveloping grasp and to move the object laterally for a distance of $300 \mathrm{~mm}$. Trials were conducted using the newly developed hand haptic interface and using the fingertip force 
display device only. The diameter and mass of the cylindrical object were $100 \mathrm{~mm}$ and $50 \mathrm{~g}$, respectively. After manipulation of the virtual object, the subjects were asked to rate the following criteria on a five-point scale, in which 1 was the lowest rating and 5 was the highest rating:

(a) Comfort while wearing the device

(b) Heaviness of the device

(c) Annoyance associated with wearing the device

(d) Existence of force feeling at the finger pad

(e) Operability

(f) Applied force consistency with virtual reality (VR)

The results of the questionnaire are shown in Fig. 10. The subjects rated the developed haptic interface as being less comfortable to wear, heavier, and more annoying to wear than the fingertip force display device only. However, the subjects rated the developed haptic interface higher in regard to the existence of force feeling at the finger pad, operability, and applied force consistency with VR as compared to the finger tip force display device only. This evaluation was the result of experiencing force display at the finger pads of the hand. We analyzed the statistical significance of evaluation items (e) and (f). A t-test with a 10\% significance level indicated no significant difference between the trials conducted using the newly developed hand haptic interface and those conducted using the fingertip force display device only for evaluation item (e), and a t-test with a $5 \%$ significance level indicated a significant difference between the trials conducted using the newly developed hand haptic interface and those conducted using the fingertip force display device only for evaluation item (f).

Subjects indicated that grasping the virtual object with an enveloping grasp when using the newly developed hand haptic interface, was easy and that the shape of the object could be sensed based on the contact force applied to the hand. Subjects pointed out that it was not easy to put on the hand haptic interface by oneself.

(a) Comfort while wearing the device

(b) Heaviness of the device

(c) Annoyance associated with wearing the device

(d) Existence of force feeling at the fingerpad

(e) Operability

(f) Applied force consistency with VR

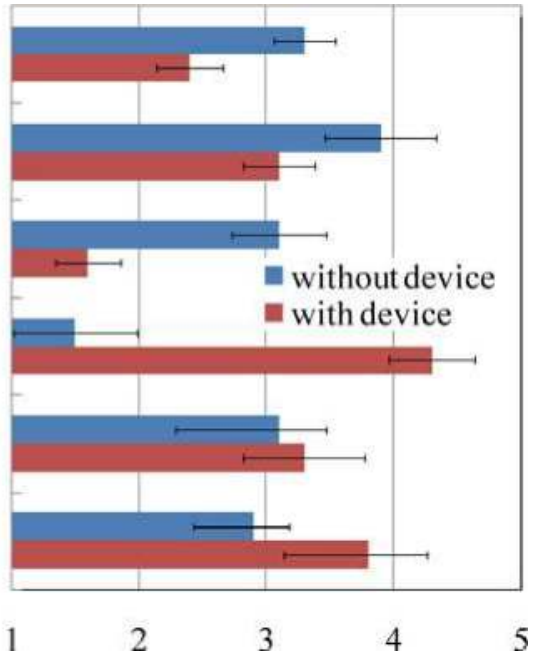

Fig. 10. Questionnaire results of cylindrical object handling in cases of with and without finger pad force display devices 


\section{B. Touching of rotating polyhedron}

We made comparative evaluations of subjects' experiences when touching a rotating polyhedron by using the index finger only for the following three cases:in case A, subjects used a HIRO II with finger pad force display on the distal and proximal interphalangeal finger pads as shown in Fig.11 (a); in case B, subjects used a HIRO II with finger pad force display on the proximal interphalangeal finger pad, as shown in Fig.11 (b); and in case C, subjects used a HIRO II without finger pad force display. The object in a virtual environment was a polyhedron with 50 plates consisting of isosceles triangles and trapezoids, as shown in Fig. 12. The polyhedron was rotating at a rate of about $3 \mathrm{rad} / \mathrm{s}$ around an axis that was orthogonal with respect to the flexion/extension axis of the index finger. After contacting the virtual object, the subjects were asked to rate the following criteria on a five-point scale, in which 1 was the lowest rating and 5 was the highest rating:

(a) Comfort while wearing the device

(b) Heaviness of the device

(c) Annoyance associated with wearing the device

(d) Existence of force feeling at the distal interphalangeal

(e) Existence of force feeling at the proximal interphalangeal

(f) Operability

(g) Consistency between force and computer graphics (CG)

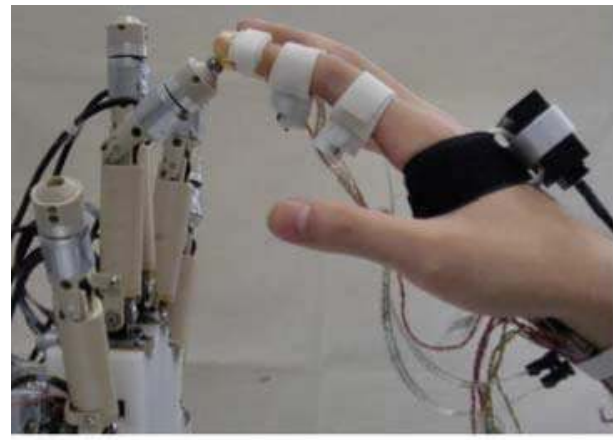

(a) Devices on distal and proximal interphalangeal finger pads

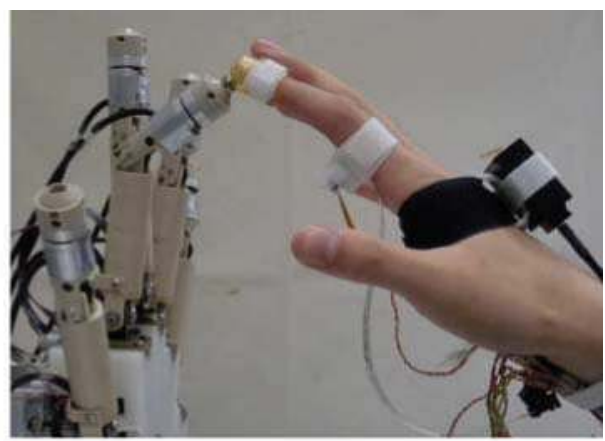

(b) Devices on proximal interphalangeal finger pad

Fig. 11. Two cases of experiment condition

The results of the questionnaire are shown in Fig. 13. Case A received the highest score in regard to the consistency between force and CG, but the lowest score in regard to the comfort, heaviness, annoyance, and operability. Case $\mathrm{C}$ received the highest score in regard to the comfort, heaviness, annoyance, and operability, but the lowest score in regard to the consistency. Case B received an intermediate score in all criteria. We analyzed the statistical significance of evaluation items a) to g). A Tukey test with a 5\% significance level indicated no significant difference between case A and case B for evaluation items (a) and (e) and between case B and case $C$ for evaluation item (d), and indicated a significant difference for the other times.

Most of the subjects mentioned that case B was better than case A for touching the rotating object, because this task was simple, it was easier to move the index finger, and the time 
needed to set up the finger pad display was smaller. These observations indicate that the finger pad force display contributes to enhancing the sense of reality in a virtual environment, but it generates a feeling of irritability in the subjects. This is a trade-off problem.

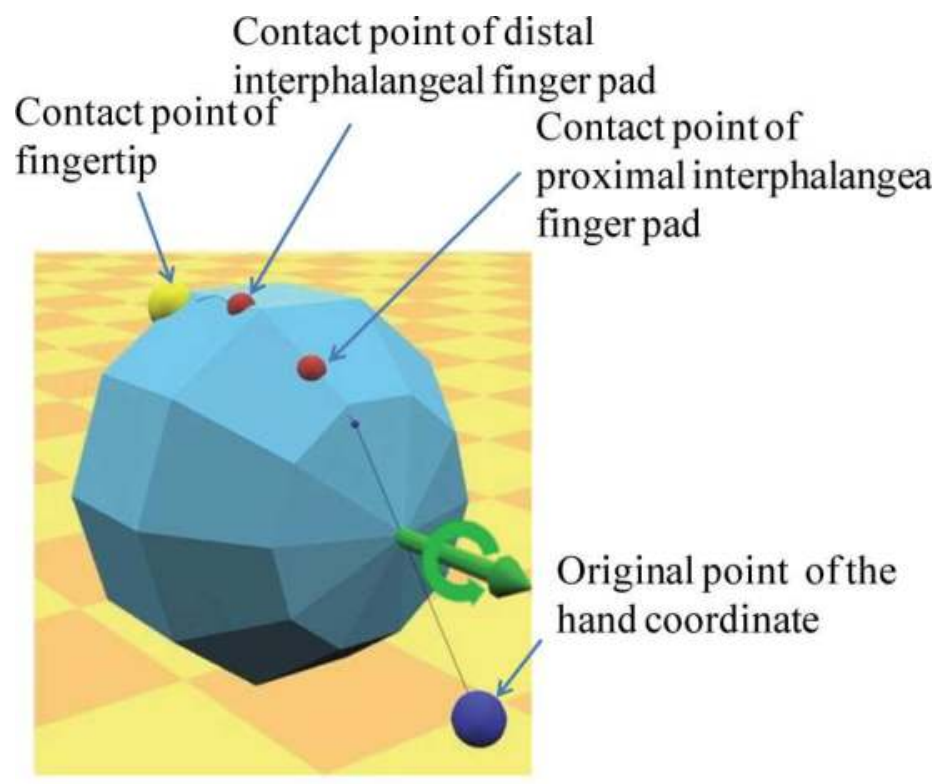

Fig. 12. Rotating polyhedron with 50 plates

\section{Conclusions}

We have presented a hand haptic interface for use in a virtual reality environment such as virtual palpation training. The hand haptic interface consists of novel finger pad force display devices and a 3D fingertip haptic interface (HIRO II). The developed finger pad force display device is driven by a flat-type brushless DC motor and can be attached at two points on the finger pads. The design concept of the hand haptic interface, the control method of the finger pad force display device, and results of an experimental evaluation have been presented. Questionnaire results revealed that the developed hand haptic interface is useful in virtual object manipulation. For a simple task such as touching a rotating polyhedron with an index finger, our evaluation showed that the use of HIRO II with one finger pad force display is better than that with two finger pad force displays to achieve both enhancement of reality in a virtual environment and reduction of discomfort when handling the haptic interface.

\section{Acknowledgments}

The present study was supported in part by the Strategic Information and Communications R\&D Promotion Program (SCOPE) of the Ministry of Internal Affairs and Communications and by a Grant-in-Aid for Scientific Research from JSPS, Japan ((B) No. 19360190). 
(a) Comfort while wearing the device

(b) Heaviness of the device

(c) Annoyance associated with wearing the device

(d) Existence of force feeling at the distal interphalangeal

(e) Existence of force feeling at the proximal interphalangeal

(f) Operability

(g) Consistency between force and CG

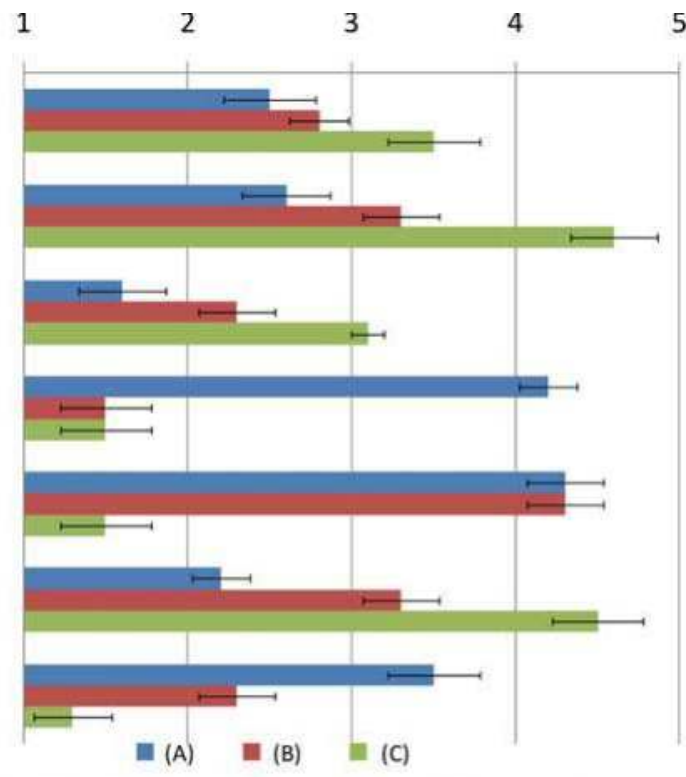
(A) Case A: with wearing device on distal and proximal interphalangeal finger pads
(B) Case B: with wearing device on proximal interphalangeal finger pad
(C) Case $\mathrm{C}$ : without wearing device

Fig. 13. Questionnaire results of rotating polyhedron object handling in three cases; Case A is HIRO II with finger pad force display devices on distal and proximal interphalangeal finger pads, Case B is HIRO II with finger pad force display devices on proximal interphalangeal finger pad, and Case C is only HIRO II.

\section{References}

[1] H. Kawasaki and T. Hayashi, Force Feedback Glove For Manipulation of Virtual Objects, Jour. of Robotics and Mechatronics, Vol. 5, No. 1, pp. 79-84, 1993.

[2] M. Bouzit, G. Burdea, G. Popescu, and R. Boian, The Rutgers Master II - New Design Force-Feedback Glove, IEEE/ASME Trans. on Mechatronics, Vol. 7, No. 2, pp. 256263, 2002.

[3] T. Koyama, K. Takemura, and T. Maeno, Development of Ultrasonic Clutch for Multi-Fingered Exoskeleton Haptic Device using Passive Force feedback for Dexterous Teleoperation, Proc. of IROS 2003, pp. 2229-2234, 2003.

[4] M. Fontana, D. Dettori, F. Salsedo, and M. Bergamasco, Mechanical Design of a Novel Hand Exoskeleton for Accurate Force Displaying, Proc. of ICRA2009, pp. 10741709, 2008.

[5] ImmersionCorporation:CyberForce, http://www.immersion.com/3d/products/cyber_force.php. 
[6] G. Inaba and K. Fujita, A Pseudo-Force-Feedback Device by Fingertip Tightening for Multi-Finger Object Manipulation, Proc. of EuroHaptics 2006.

[7] Y. Ueda and T. Maeno, Development of a Mouse-Shaped Haptic Device with Multiple Finger Inputs, Proc. of IROS2004, pp. 2886-2891(2004).

[8] T. Yoshikawa and A. Nagara, Development and Control of Touch and Force Display Devices for Haptic Interface, Proc. of SYROCO'00, pp. 427-432, 2000.

[9] Y. Adachi, et al., Development of a Haptic Device for Multi Fingers by Macro-Micro Structure, Journal of the Robotics Society of Japan, Vol. 20, No. 7, pp. 725-733, 2002.

[10] S. Walairacht, M. Ishii, Y. Koike, and M. Sato, Two-Handed Multi-Fingers String-Based Haptic Interface Device, IEICE Trans. on Information and Systems, Vol. E84D, No. 3, pp. 365-373, 2001.

[11] M. Montoy, M. Oyarzabal, M. Ferre, A. Campos, and J. Barrio, MasterFinger: Multifinger Haptic Interface for Collaborative Environment, Proc. of EuroHaptics 2008, pp. 411-419, 2008.

[12] H. Kawasaki and T. Mouri, Design and Control of Five-Fingered Haptic Interface Opposite to Human Hand, IEEE Transaction on Robotics, Vol. 23, No. 5, October, pp. 909-918, 2007.

[13] M. O. Alharabi, V. Daniulaitis, H. Kawasaki, and T. Hori, Medical Training Simulation for Palpation of Subsurface Tumor Using HIRO, Proc. WorldHaptics2005, Pisa, pp. 623-624, 2005.

[14] S. J. Lederman, Skin and Touch, Encyclopedia of Human Biology, Vol. 7, pp. 51-63, 1991.

[15] T. Endo, H. Kawasaki, T. Kanno and T. Mouri, Perception of the Force Direction and Skill Transfer by using Five-Fingered Haptic Interface, Proceedings of the 13th International Conference on Virtual Systems and Multimedia (VSMM'07), Brisbane, 2007

[16] T. Grieve, Y. Sun, J. M. Hollerbach, and S. A. Mascaro, 3-D Force Control on the Human Fingerpad Using a Magnetic Levitation Device for Fingernail Imaging Calibration, Proc. of 3rd Joint Eurohaptics Conference and Symposium on Haptic interfaces for Virtual Environment and Teleoperator System, pp. 411-416, 2009.

[17] K. Minamizawa, S. Fukamachi, H. Kajimoto, N. Kawakami, and S. Tachi, Wearable Haptic Display to Present Mass and internal Dynamics of Virtual Objects, TVRSJ, Vol. 13, No. 1, 2008.

[18] T. Aoki, H. Mitake, S. Hasegawa, and M. Sato, Wearable Haptic Device to Present Contact Sensation, Based on Cutaneous Sensation using Thin Wires, TVRSJ Vol. 14, No. 3, pp. 421-428, 2009.

[19] I. M. Koo, K. Jung, J. C. Koo, J. Nam, and Y. K. Lee, Development of Soft-Actuator Based Wearable Tactile Display, IEEE Trans. on Robotics, Vol. 24, No. 3, pp. 548$558,2008$.

[20] WEB of Exseal Corporation: http://www.exseal.net/. 
[21] H. Kawasaki and T. Komatsu, Mechanism Design of Anthropomorphic Robot: Gifu Hand I, Journal of Robotics and Mechatronics, Vol. 11, No. 4, pp. 269-273, 1999. 


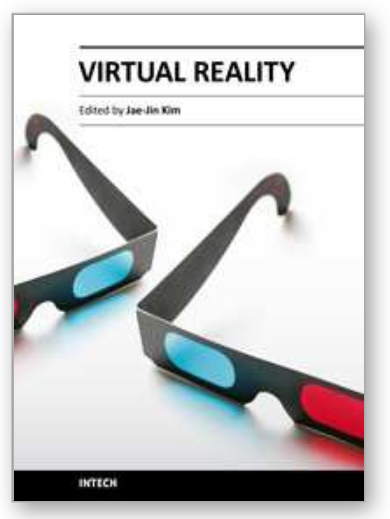

\author{
Virtual Reality \\ Edited by Prof. Jae-Jin Kim
}

ISBN 978-953-307-518-1

Hard cover, 684 pages

Publisher InTech

Published online 08, December, 2010

Published in print edition December, 2010

Technological advancement in graphics and other human motion tracking hardware has promoted pushing "virtual reality" closer to "reality" and thus usage of virtual reality has been extended to various fields. The most typical fields for the application of virtual reality are medicine and engineering. The reviews in this book describe the latest virtual reality-related knowledge in these two fields such as: advanced human-computer interaction and virtual reality technologies, evaluation tools for cognition and behavior, medical and surgical treatment, neuroscience and neuro-rehabilitation, assistant tools for overcoming mental illnesses, educational and industrial uses In addition, the considerations for virtual worlds in human society are discussed. This book will serve as a state-of-the-art resource for researchers who are interested in developing a beneficial technology for human society.

\title{
How to reference
}

In order to correctly reference this scholarly work, feel free to copy and paste the following:

Haruhisa Kawasaki, Shinya Koide, Tetuya Mouri and Takahiro Endo (2010). Development of a Finger Pad Force Display for a Hand Haptic Interface, Virtual Reality, Prof. Jae-Jin Kim (Ed.), ISBN: 978-953-307-518-1, InTech, Available from: http://www.intechopen.com/books/virtual-reality/development-of-a-finger-pad-forcedisplay-for-a-hand-haptic-interface

\section{INTECH}

open science | open minds

\author{
InTech Europe \\ University Campus STeP Ri \\ Slavka Krautzeka 83/A \\ 51000 Rijeka, Croatia \\ Phone: +385 (51) 770447 \\ Fax: +385 (51) 686166 \\ www.intechopen.com
}

\author{
InTech China \\ Unit 405, Office Block, Hotel Equatorial Shanghai \\ No.65, Yan An Road (West), Shanghai, 200040, China \\ 中国上海市延安西路65号上海国际贵都大饭店办公楼 405 单元 \\ Phone: +86-21-62489820 \\ Fax: +86-21-62489821
}


(C) 2011 The Author(s). Licensee IntechOpen. This chapter is distributed under the terms of the Creative Commons Attribution-NonCommercialShareAlike-3.0 License, which permits use, distribution and reproduction for non-commercial purposes, provided the original is properly cited and derivative works building on this content are distributed under the same license. 